\title{
A Vehicle Routing Problem with Consideration of Green Transportation
}

\author{
Che-Fu Hsueh ${ }^{1}$ \\ ${ }^{1}$ Department of Marketing and Distribution Management, Chien Hsin University of Science and Technology, \\ Taoyuan, Taiwan \\ Correspondence: Che-Fu Hsueh, Department of Marketing and Distribution Management, Chien Hsin University \\ of Science and Technology, Taoyuan, Taiwan. E-mail: cfhsueh@uch.edu.tw
}

$\begin{aligned} & \text { Received: October 16, } 2017 \\ & \text { Accepted: October 30, } 2017 \quad \text { Online Published: November 2, } 2017 \\ & \text { doi:10.5539/jms.v7n4p89 }\end{aligned}$ URL: http://doi.org/10.5539/jms.v7n4p89

\begin{abstract}
This study aims to investigate the Green Vehicle Routing Problem (GVRP), which considers stochastic traffic speeds, so that fuel consumption and emissions can be reduced. Considering a heterogeneous fleet, the fuel consumption rate differs due to several factors, such as vehicle types and conditions, travel speeds, roadway gradients, and payloads. A mathematical model was proposed to deal with the GVRP, and its objective is to minimize the sum of the fixed costs and the expected fuel consumption costs. A customized genetic algorithm was proposed for solving the model. The computational experiments confirm the efficiency of the algorithm and show that the solution of GVRP is quite different from that of the traditional vehicle routing problem. We suggest that a company should use light vehicles to service the customers situated at higher terrains. The customers with higher demands can be visited earlier, but the customers situated at higher terrains or far away from the depot should be visited later. The study also found that the fixed costs of dispatching vehicles are critical in GVRP; a logistics company may thus tend to use large vehicles, despite that it may cause higher fuel consumption and emissions. The proposed model and algorithm are capable of suggesting a guidance for green logistics service providers to adopt a beneficial vehicle routing plan so as to eventually achieve a low economic and environmental cost.
\end{abstract}

Keywords: vehicle routing problem, green transportation, genetic algorithm, fuel consumption, emission

\section{Introduction}

In recent years, with the continued trend of environmental protection and sustainable development, the issues of green supply chain management, green logistics, and green transportation have received increasing and close attention from business organizations, academics, and governments. One of the most important and urgent issues is to reduce fuel consumption and $\mathrm{CO} 2$ emissions, especially caused by transportation activities.

In the traditional Vehicle Routing Problem (VRP), a common objective is to minimize the total travel distance (or travel time) of all vehicles. Such an objective, however, does not always result in less fuel consumptions or $\mathrm{CO} 2$ emissions. Many factors will affect fuel consumptions and emissions, such as vehicle types, payloads, roadway gradients, pavement conditions, and travel speeds. Among these factors, the travel speed has much higher uncertainty due to dynamic traffic conditions, especially in urban areas. The studies about stochastic travel speed in green VRP (GVRP) are not commonly seen in recent literature.

The literatures of GVRP can be approximately divided into energy consumption, pollution emissions and reverse logistics. To match this research subject and requirements, the literatures about energy consumption and pollution emissions are reviewed and discussed below.

At present, almost all vehicles of the third-party logistics, transport companies, even enterprises' fleets use fossil fuel as power source. The fuel cost is an important part of transportation cost (Xiao, Zhao, Kaku, \& Xu, 2012). The transportation activity is one of the main sources of greenhouse gas emission. The traditional vehicle routing planning which only considers the distance cannot actually minimize the greenhouse gas emission, because the factors influencing the emission also include speeds, loads and so on. Reducing the fuel consumption is the most direct objective of GVRP. In addition, the emissions of greenhouse gases can be derived directly from the fuel consumption to measure the influence on the environment (Mohammadi, Torabi, \& Tavakkoli-Moghaddam, 2014). Demir, Bektas, \& Laporte (2014) classified the factors influencing fuel consumption into five major 
classes: vehicle, environment, traffic, driving and operation. Most literatures only discussed some of these factors.

Kara, Kara, \& Yetis (2007) brought distance and load into the cost function of a capacitated VRP. However, the cost function lacks further measurement of fuel consumption or emission. Kuo (2010) dealt with a time-dependent VRP, which considers distance, speed and load simultaneously for fuel consumption, and guaranteed the vehicle's First-In-First-Out constraint. However, the paper did not discuss the relationship between speed and fuel consumption rate. Xiao et al. (2012) assumed the fuel consumption rate be a linear function of loads, and built an integer programming model containing fixed cost and fuel cost. Demir, Bektas, \& Laporte (2012) considered the fuel cost, $\mathrm{CO} 2$ emission cost and driver's salary in the objective equation, and proposed an integer linear programming model along with a heuristic algorithm to deal with the Pollution-Routing Problem.

Recently, Qian, \& Eglese (2014) discussed the problem of finding a route and schedule for a vehicle in a time-dependent network where the objective is to minimize the greenhouse gas emissions, and proposed two solution methods: dynamic programming and heuristic algorithm. Felipe, Ortuño, Righini, \& Tirado (2014) presented several heuristics for a variation of the vehicle routing problem in which the transportation fleet is composed of electric vehicles with limited autonomy in need for recharge during their duties. However, as the nonelectric vehicles were not considered in their study, the fuel consumption and pollution emissions were also disregarded; the objective function only considered recharging costs and battery costs. Tajik, Tavakkoli-Moghaddam, Vahdani, \& Mousavi (2014) built a mixed integer linear programming model for the VRP with pickup and delivery; the objectives considered the fuel cost, $\mathrm{CO} 2$ emission cost, driver cost and time window violation cost. Tiwari \& Chang (2015) used clustering and genetic algorithms to solve GVRP. The objective function considered the minimum distance travelled by each vehicle as well as the total emitted $\mathrm{CO} 2$, which was directly assumed to be the product of mean distance, load and conversion factor. Soysal, Bloemhof-Ruwaard, \& Bektas (2015) considered a delivery network with a central station, several satellite stations and many customer points. Time-dependent travel times were considered. The objective function considered fuel costs, station costs and driver costs. The method of calculating fuel consumption is similar to that of Demir et al. (2012). Demir et al. (2014) and Lin, Choy, Ho, Chung, \& Lam (2014) reviewed many studies for GVRP, and suggested some research directions. Their studies help to rapidly understand the development of GVRP.

\section{Model Formulation}

\subsection{Notations}

Sets:

K set of vehicles

$N \quad$ set of customers

$N_{0} \quad$ set of the depot and customers

$S_{i j} \quad$ set of segments in the path $(i, j)$

Decision variables:

$x_{i j k} \quad$ binary variable $; 1$ if vehicle $k$ travels from node $i$ to node $j$ and 0 otherwise

$q_{i j} \quad$ payloads between node $i$ and node $j$

Functions and parameters:

$\tilde{\rho}_{i j k s} \quad$ fuel consumption rate, which is a function of speed, load, and roadway gradients

$F C_{k} \quad$ fixed cost of dispatching vehicle $\mathrm{k}$

$c \quad$ unit cost of fuel consumptions and emissions

$d_{i j s} \quad$ length of segment $s$ in the $\operatorname{arc}(i, j)$

$Q_{k} \quad$ capacity of vehicle $k$

$D_{i} \quad$ demand of customer $i$

\subsection{Problem Description}

The GVRP is defined on a complete graph, where vertex set $N_{0}$ is a combination of the customer set $\mathrm{N}$ and the depot. The edges of the graph represent the paths between vertexes. An edge or path is further divided into several segments according to their different gradients, speeds, or other road conditions that will affect fuel 
consumptions. Among a heterogeneous vehicle set $\mathrm{K}$, a number of vehicles depart from the depot, visit customers for delivery, and return to the depot. A customer with certain demands can be visited exactly once. Due to uncertain traffic conditions, the speed on a segment is assumed to be a random variable. The fuel consumption rate on a segment, which is also random, is assumed to be a function of the gradient, speed, and payloads on that segment. The GVRP seeks to find at most $|K|$ vehicle routes which start and end at the depot, such that the total cost, including fixed costs of dispatching vehicles and expected costs of fuel consumptions and emissions, is minimized.

\subsection{Mathematical Model}

Based on the above problem description, the GVRP is formulated as follows:

$$
\begin{gathered}
\text { Min TC }=\sum_{k \in K}\left(F C_{k} \sum_{j \in N} x_{0 j k}\right)+c \sum_{i, j \in N_{0}, i \neq j} \sum_{k \in K} \sum_{s \in S_{i j}} d_{i j s} E\left[\tilde{\rho}_{i j k s}\right] x_{i j k} \\
\text { Subject to: } \sum_{j \in N} x_{0 j k} \leq 1 \quad \forall k \in K \\
\sum_{i \in N_{0}, i \neq j} \sum_{k \in K} x_{i j k}=1 \quad \forall j \in N \\
\sum_{j \in N_{0}, j \neq i} x_{j i k}-\sum_{j \in N_{0}, j \neq i} x_{i j k}=0 \quad \forall i \in N, k \in K \\
\sum_{j \in N_{0}, j \neq i} q_{j i}-\sum_{j \in N_{0}, j \neq i} q_{i j}=D_{i} \quad \forall i \in N \\
q_{i j} \leq \sum_{k \in K} Q_{k} x_{i j k} \quad \forall i, j \in N_{0} \\
x_{i j k} \in\{0,1\} \quad \forall i, j \in N_{0}, i \neq j, k \in K \\
q_{i j} \geq 0 \quad \forall i, j \in N_{0}, i \neq j
\end{gathered}
$$

The objective function represented by equation (1) is to minimize the total cost, including fixed costs of dispatching vehicles, and expected costs of fuel consumptions and emissions. Constraint (2) requires each vehicle depart depot at most once. Constraints (3) and (4) require each customer be serviced exactly once by a certain vehicle. Constraint (5) defines the demand as the difference of payloads before and after service. Note that Constraints (5) also eliminates sub-tours which do not connect to the depot. The vehicle capacity constraint is presented in (6). Constraint (7) defines $x_{i j k}$ as a binary variable, and Constraint (8) ensures the non-negativity of the loads en route.

\section{Solution Algorithm}

In the following, we propose a genetic algorithm to solve the GVRP model presented in the previous section. After introducing the method of chromosome encoding, the steps of the algorithm include: population initialization, fitness evaluation, selection, crossover, and mutation.

\subsection{Chromosome Encoding}

A solution of GVRP can be represented as a two-part chromosome; Chromosome-I is a sequence of customers, and Chromosome-II identifies the index of the last-serviced customer in Chromosome-I for each vehicle. The length of Chromosome-I equals the number of customers, while the length of Chromosome-II equals the number of vehicles. Each element in Chromosome-I and Chromosome-II is regarded as a gene. Figure 1 illustrates the chromosome encoding for a solution. For example, there are 12 customers to be serviced by 4 vehicles. Figure 1 shows that the solution consists of three routes: $0-7-3-4-0,0-12-1-8-2-0$, and $0-11-5-6-10-9-0$, assigned to Vehicle 1, Vehicle 2, and Vehicle 4, respectively. Note that " 0 " represents the depot and Vehicle 3 is not used.

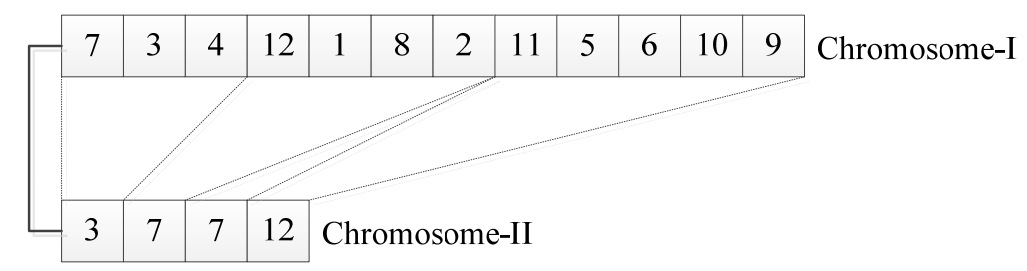

Figure 1. Example of chromosome encoding

\subsection{Population Initialization}

Initial feasible solutions are generated repeatedly until the population size is reached. For each solution, Chromosome-I is generated randomly. After that, each vehicle (i.e., a gene in Chromosome-II) can be chosen to 
service customers or not, randomly. Once a vehicle is chosen, it will service successive customers in Chromosome-I as many as possible subject to the capacity constraint. The process for handling Chromosome-II may repeat until all customers are assigned to a vehicle.

\subsection{Fitness Evaluation}

The fitness function that minimizes the total cost, including fixed costs of dispatching vehicles, and expected costs of fuel consumptions and emissions in the GVRP model is shown in Eq. (1).

\subsection{Selection}

Selection phase is the process where the individuals are selected based on their fitness to mate and produce new offspring. In this study we use the tournament method, where the numbers of randomly picked individuals compete in the tournament. The winner of the tournament and thus the selected individual is always the fittest individual from the tournament.

\subsection{Crossover}

Crossover is the process that mimics mating between two individuals with the goal of producing children. As in nature, those children take characteristics from both parents. In this study, the crossover only applies to Chromosome-I. We use two-point partially matched crossover method to produce two children at a time, as illustrated in Figure 2. Choose two random points in parent chromosomes and exchange the genes in the middle part. The resulting duplicate genes outside the exchanging part are further exchanged based on the mapping set, which is constructed by comparing the exchanging parts of two parents.
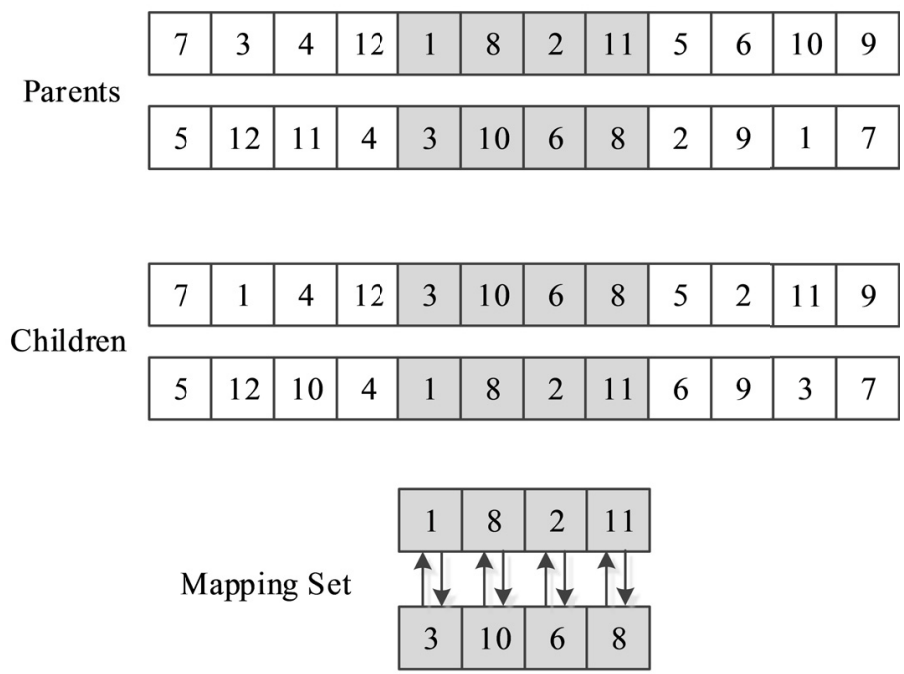

Figure 2. Example of crossover

After the Chromosome-I of the children are generated, the Chromosome-II of the children are modified if necessary to fulfill the capacity constraint, ensuring the feasibility of the offspring.

\subsection{Mutation}

Mutation is an operator that serves as the mean to widen the search space for a small degree. In nature the mutation has a small chance of happening and the same is in the GA. In this study, we adopt two mutation methods to search solutions in a wider space, i.e., exchange mutation and insertion mutation. Exchange mutation simply exchange two random-selected genes in Chromosome-I. Insertion mutation removes a random-selected gene from its place and inserts it in a different position. After mutation is performed, the Chromosome-II is modified again if necessary for feasibility.

\section{Numerical Examples}

\subsection{Data Set}

The experiments are conducted for three different kinds of trucks. The information of the trucks is listed in Table 1, in which some data are derived from Mitsubishi Fuso Truck and Bus Corporation, while others are borrowed from Demir et al. (2012). The fleet consists of two Type 1 trucks, three Type 2 trucks, and five Type 3 trucks. 
For a road segment, according to Demir et al. (2012), the fuel consumption rate function is expressed as follows:

$$
\tilde{\rho}_{i j k s}=\frac{\xi}{K \psi}\left(\frac{k N V}{v}+\gamma(w+f)\left(g \sin \theta+g C_{r} \cos \theta\right)+\beta \gamma v^{2}\right)
$$

where $v$ is a random speed, $f$ is payload, $\theta$ is the radian of road angle, and other parameters are also summarized in Table 1.

Table 1. Information of trucks and other parameters

\begin{tabular}{|c|c|c|c|c|}
\hline Notation & Description & $\begin{array}{l}\text { Type } 1 \\
\text { (large size) }\end{array}$ & $\begin{array}{l}\text { Type } 2 \\
\text { (median size) }\end{array}$ & $\begin{array}{l}\text { Type } 3 \\
\text { (small size) }\end{array}$ \\
\hline$w$ & Curb weight $(\mathrm{Kg})$ & 3900 & 2570 & 2400 \\
\hline V & Engine displacement (liters) & 7.545 & 2.998 & 2.998 \\
\hline$N$ & Engine speed (rev/second) & 33 & 33 & 33 \\
\hline$k$ & Engine friction factor (kilojoule/rev/liter) & 0.2 & 0.2 & 0.2 \\
\hline$A$ & Frontal surface area $\left(\mathrm{m}^{2}\right)$ & 5.54 & 4.47 & 3.89 \\
\hline$Q_{k}$ & Load capacity $\left(m^{3}\right)$ & 29 & 17 & 9.5 \\
\hline$F C_{k}$ & Fixed cost (dollars) & 60 & 55 & 50 \\
\hline Notation & Description & & Value & \\
\hline$\xi$ & Fuel-to-air mass ratio & & 1 & \\
\hline$K$ & Heating value of a typical diesel fuel (kilojoule/gram) & & 44 & \\
\hline$\psi$ & Conversion factor (gram/second to liter/second) & & 737 & \\
\hline$\beta$ & Constant & & $0.5 C_{d} \rho A$ & \\
\hline$C_{d}$ & Coefficient of aerodynamic drag & & 0.7 & \\
\hline$\rho$ & Air density (kilogram/meter3) & & 1.2041 & \\
\hline$\gamma$ & Constant & & $1 / 360$ & \\
\hline$g$ & Gravitational constant (meter/second ${ }^{2}$ ) & & 9.81 & \\
\hline$C_{r}$ & Coefficient of rolling resistance & & 0.01 & \\
\hline
\end{tabular}

Forty customers and a depot are scattered on a $20 \mathrm{~km} \times 20 \mathrm{~km}$ area, which is not totally plain. Figure 3 shows the terrain of the area as well as the location of the customers and the depot. The darker area refers to a higher elevation. The depot is located at $(10,3)$. Customers 1 to 22 are randomly scattered on the rectangular area $(5,5)$ - $(15,15)$, which is assumed to be the downtown. Customers 23 to 28 are randomly scattered on the rectangular area $(0,0)-(5,20)$. Customers 29 to 34 are randomly scattered on the rectangular area $(15,0)-(20,20)$. Customers 35 to 37 are randomly scattered on the rectangular area $(5,15)-(15,20)$. Customers 38 to 40 are randomly scattered on the rectangular area $(5,0)-(15,5)$. The volumes and weights of the demands and the coordinates of the customers are listed in Table 2.

The distance between nodes is assumed to be block distances; the vehicle moves only along the path parallel to the $\mathrm{x}$ axis or the $\mathrm{y}$ axis. The path is divided into several segments according to different gradients, speeds, or other road conditions that will affect fuel consumptions. For each segment, the random speed is characterized by the following probability density function, which is illustrated in Figure 4:

$$
f(v)=\left\{\begin{array}{cc}
2 /(2 b-a-c) & \text { if } c \leq v \leq b \\
(2 /(2 b-a-c))((v-a) /(c-a)) & \text { if } a \leq v \leq c \\
0 & \text { else }
\end{array}\right.
$$

In this study we assume that truck drivers always drive under the speed limit. The travel speed is stochastic and may be lowered due to traffic conditions. Although the above probability density function is only an example, it does characterize the travel speed properly. 
Table 2. Demands of the customers

\begin{tabular}{|c|c|c|c|c|c|c|c|c|c|c|}
\hline Customer & 1 & 2 & 3 & 4 & 5 & 6 & 7 & 8 & 9 & 10 \\
\hline Volume $\left(\mathrm{m}^{\wedge} 3\right)$ & 3.68 & 0.40 & 2.70 & 2.37 & 2.52 & 3.77 & 3.83 & 3.32 & 0.62 & 0.12 \\
\hline Weight (Kg) & 352 & 44 & 254 & 269 & 225 & 350 & 391 & 290 & 69 & 15 \\
\hline Coordinate $\mathrm{x}$ & 14056 & 8672 & 13458 & 6870 & 6703 & 9032 & 5742 & 6562 & 8448 & 13148 \\
\hline Coordinate y & 14074 & 6611 & 8644 & 10087 & 13299 & 8303 & 14686 & 14781 & 12265 & 6772 \\
\hline Customer & 11 & 12 & 13 & 14 & 15 & 16 & 17 & 18 & 19 & 20 \\
\hline Volume $\left(\mathrm{m}^{\wedge} 3\right)$ & 0.42 & 0.93 & 2.39 & 3.95 & 2.36 & 0.26 & 1.90 & 1.90 & 3.77 & 1.24 \\
\hline Weight (Kg) & 37 & 78 & 205 & 469 & 219 & 27 & 227 & 223 & 331 & 119 \\
\hline Coordinate $\mathrm{x}$ & 11055 & 10446 & 13525 & 7307 & 12118 & 8921 & 14199 & 7301 & 6319 & 8310 \\
\hline Coordinate y & 8227 & 14529 & 11479 & 13845 & 11730 & 10502 & 11548 & 10998 & 9802 & 13815 \\
\hline Customer & 21 & 22 & 23 & 24 & 25 & 26 & 27 & 28 & 29 & 30 \\
\hline Volume $\left(\mathrm{m}^{\wedge} 3\right)$ & 3.68 & 3.38 & 3.54 & 3.19 & 0.81 & 2.65 & 3.95 & 3.14 & 2.85 & 0.34 \\
\hline Weight (Kg) & 440 & 332 & 410 & 317 & 95 & 266 & 462 & 273 & 275 & 31 \\
\hline Coordinate $\mathrm{x}$ & 10623 & 7403 & 4721 & 761 & 4598 & 1436 & 3175 & 1773 & 19348 & 16615 \\
\hline Coordinate y & 9728 & 5644 & 158 & 3173 & 18048 & 6108 & 17182 & 19188 & 7836 & 16731 \\
\hline Customer & 31 & 32 & 33 & 34 & 35 & 36 & 37 & 38 & 39 & 40 \\
\hline Volume $\left(\mathrm{m}^{\wedge} 3\right)$ & 3.39 & 2.58 & 2.23 & 0.91 & 2.40 & 2.06 & 1.35 & 3.86 & 0.10 & 0.56 \\
\hline Weight (Kg) & 388 & 261 & 215 & 91 & 272 & 199 & 127 & 402 & 10 & 57 \\
\hline Coordinate $\mathrm{x}$ & 16394 & 18043 & 17231 & 18681 & 6545 & 7881 & 12132 & 6435 & 13774 & 6424 \\
\hline Coordinate y & 19144 & 7163 & 6081 & 10487 & 16326 & 19117 & 17542 & 992 & 4034 & 4107 \\
\hline
\end{tabular}

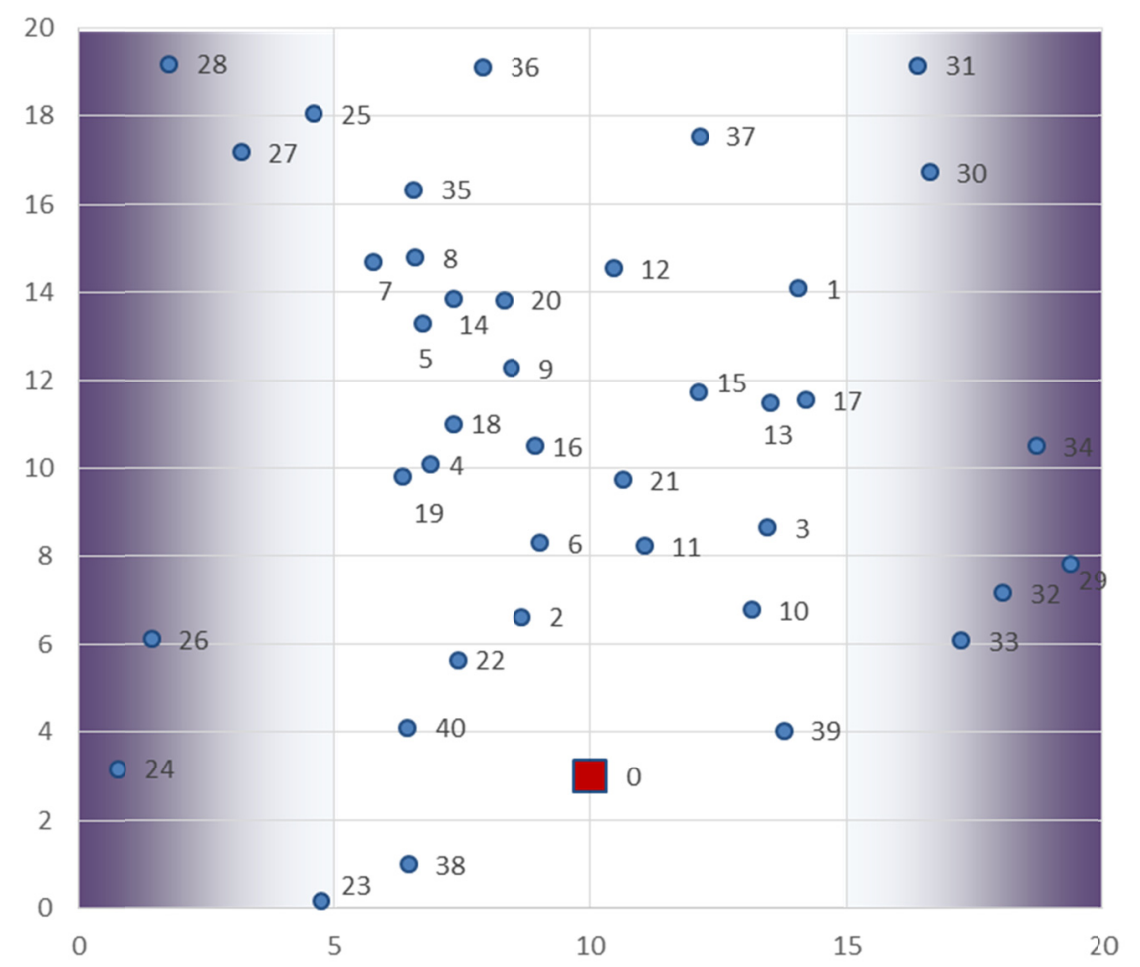

Figure 3. Location of the customers and the depot 


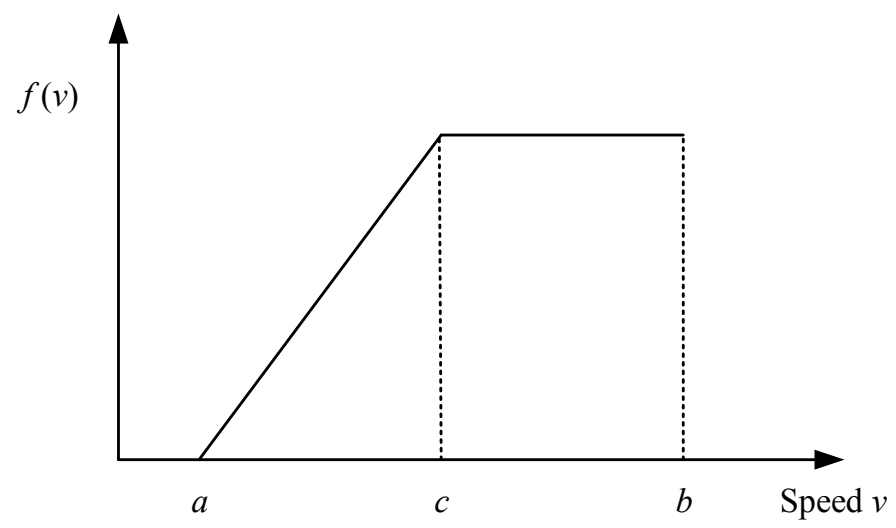

Figure 4. Probability density function of speeds

\subsection{Results and Discussions}

The tests were carried out based on the above information. First, we considered both fixed costs and expected fuel consumptions costs, as in Eq. (1). The test result shows that the fixed costs of dispatching vehicles, such as wages of drivers, dominate the solution. Only two Type-1 vehicles and one Type- 2 vehicle are dispatched to service the customers, because fewer vehicles refer to lower fixed costs. In such cases, the costs of fuel consumptions and emissions may not receive enough attentions when making decisions of vehicle routing. Therefore, reducing fixed costs is a critical issue for a green logistics company. In the second part, we performed further tests with no fixed costs to show the influence of vehicle routings on the expected costs of fuel consumptions and emissions. The test results are shown in Figure 5. Note that the links connected to the depot for each route are not shown in the figure for simplicity. The test result shows that all Type- 2 and Type- 3 vehicles are dispatched while no Type-1 vehicle is used, since heavy vehicles with heavy loads refer to more fuel consumptions and emissions. Most customers at higher terrains or at the nearby area are serviced by light vehicles (Type-3).

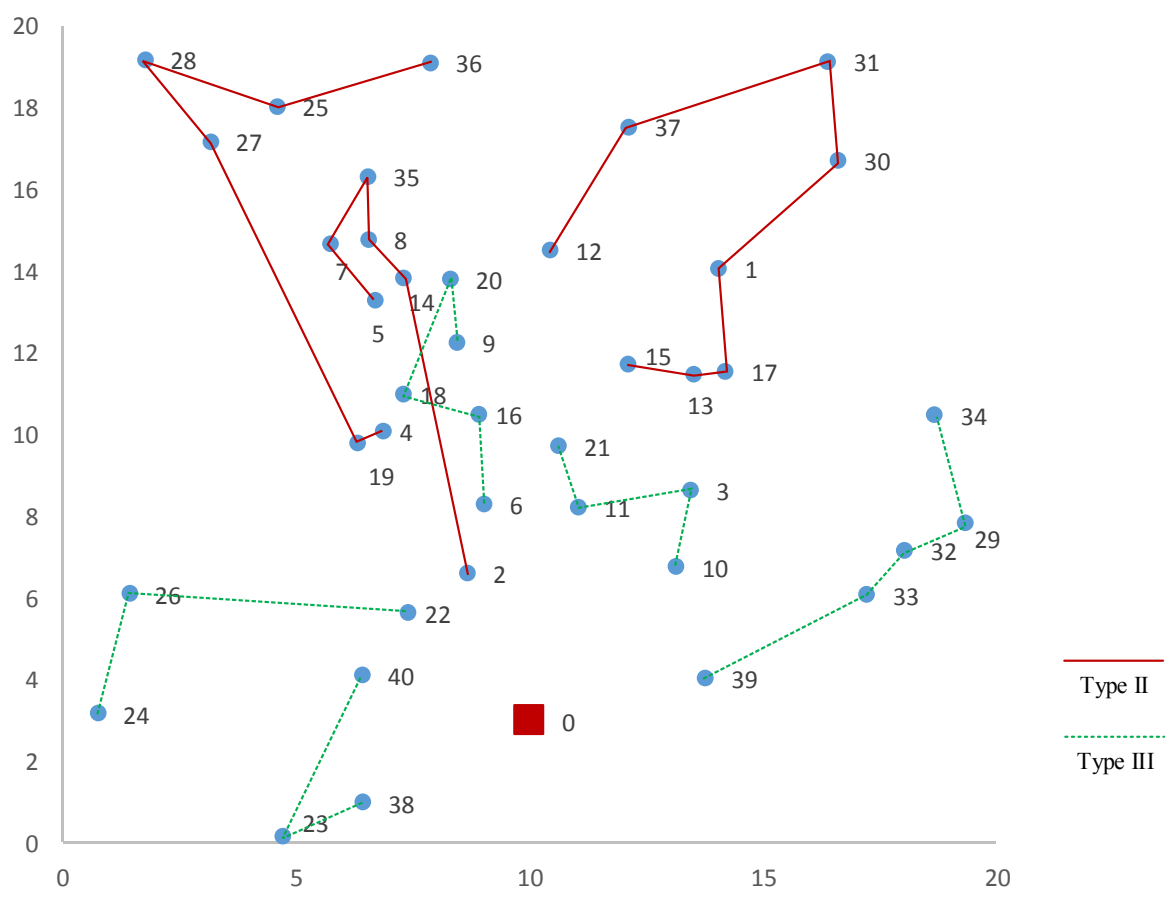

Figure 5. Computational results 


\section{Conclusion}

In this study, we proposed a mathematical model to deal with the GVRP, whose objective is to minimize the sum of the fixed costs and the expected fuel consumption costs. However, we found that if the fixed costs, especially the wages of the drivers, are considered in GVRP, the vehicle routes will be composed of large vehicles, despite that it may cause higher fuel consumption and emissions. This issue is critical especially when the transportation operation is outsourced to a third-party logistics. To deal with this issue, on the one hand the company can use advanced logistics technology to reduce fixed costs, and on the other hand more efforts should be made to reduce fuel consumption and emissions, such as internalizing the external cost of emissions, adding a constraint of emission limitation, or using more green vehicles. We suggest that a company should use light vehicles to service the customers at higher terrains. The customers with higher demands can be visited earlier, but the customers situated at higher terrains or far away from the depot should be visited later.

A genetic algorithm is used to solve the GVRP, in which a new coding method is proposed to generate a two-part chromosome. It is much easier to perform a GA operation and maintain the feasibility by using a two-part chromosome. This kind of structure can be applied to other type of VRPs.

\section{Acknowledgments}

The author would like to thank the Ministry of Science and Technology of Taiwan for its financial support. (Grant: MOST 104-2410-H-231-008-MY2)

\section{References}

Demir, E., Bektas, T., \& Laporte, G. (2012). An adaptive large neighborhood search heuristic for the Pollution-Routing Problem. European Journal of Operational Research, 223(2), 346-359. https://doi.org/10.1016/j.ejor.2012.06.044

Demir, E., Bektas, T., \& Laporte, G. (2014). A review of recent research on green road freight transportation. European Journal of Operational Research, 237(3), 775-793. https://doi.org/10.1016/j.ejor.2013.12.033

Felipe, Á., Ortuño, M. T., Righini, G., \& Tirado, G. (2014). A heuristic approach for the green vehicle routing problem with multiple technologies and partial recharges. Transportation Research Part E: Logistics and Transportation Review, 71(0), 111-128. https://doi.org/10.1016/j.tre.2014.09.003

Kara, İ., Kara, B., \& Yetis, M. K. (2007). Energy Minimizing Vehicle Routing Problem. In A. Dress, Y. Xu, \& B. Zhu (Eds.), Combinatorial Optimization and Applications (Vol. 4616, pp. 62-71). Berlin Heidelberg: Springer. https://doi.org/10.1007/978-3-540-73556-4_9

Kuo, Y. (2010). Using simulated annealing to minimize fuel consumption for the time-dependent vehicle routing problem. Computers \& Industrial Engineering, 59(1), 157-165. https://doi.org/10.1016/j.cie.2010.03.012

Lin, C., Choy, K. L., Ho, G. T. S., Chung, S. H., \& Lam, H. Y. (2014). Survey of Green Vehicle Routing Problem: Past and future trends. Expert Systems with Applications, 41(4, Part 1), 1118-1138. https://doi.org/10.1016/j.eswa.2013.07.107

Mohammadi, M., Torabi, S. A., \& Tavakkoli-Moghaddam, R. (2014). Sustainable hub location under mixed uncertainty. Transportation Research Part E-Logistics and Transportation Review, 62, 89-115. https://doi.org/10.1016/j.tre.2013.12.005

Qian, J. N., \& Eglese, R. (2014). Finding Least Fuel Emission Paths in a Network with Time-Varying Speeds. Networks, 63(1), 96-106. https://doi.org/10.1002/net.21524

Soysal, M., Bloemhof-Ruwaard, J. M., \& Bektaş, T. (2015). The time-dependent two-echelon capacitated vehicle routing problem with environmental considerations. International Journal of Production Economics, 164, 366-378. https://doi.org/10.1016/j.ijpe.2014.11.016

Tajik, N., Tavakkoli-Moghaddam, R., Vahdani, B., \& Mousavi, S. M. (2014). A robust optimization approach for pollution routing problem with pickup and delivery under uncertainty. Journal of Manufacturing Systems, 33(2), 277-286. https://doi.org/10.1016/j.jmsy.2013.12.009

Tiwari, A., \& Chang, P. C. (2015). A block recombination approach to solve green vehicle routing problem. International Journal of Production Economics, 164, 379-387. https://doi.org/10.1016/j.ijpe.2014.11.003

Xiao, Y., Zhao, Q., Kaku, I., \& Xu, Y. (2012). Development of a fuel consumption optimization model for the capacitated vehicle routing problem. Computers \& Operations Research, 39(7), 1419-1431. https://doi.org/10.1016/j.cor.2011.08.013 


\section{Copyrights}

Copyright for this article is retained by the author(s), with first publication rights granted to the journal.

This is an open-access article distributed under the terms and conditions of the Creative Commons Attribution license (http://creativecommons.org/licenses/by/4.0/). 